\title{
New Method for Characterizing the Injected-carrier-to-photon Conversion Efficiencies Inside the Ultrafast all-optical Semiconductor Gates
}

\author{
Jun Sakaguchi, Yoshiyasu Ueno, \\ University of Electro-Communications, Department of Electronics Engineering, 1-5-1, Chohugaoka, Chohu, Tokyo 182-8585, Japan, \\ sakaguchi@ultrafast.ee.uec.ac.jp
}

Kohsuke Nishimura, Tomonori Yazaki

KDDI R\&D Laboratories Inc., 2-1-15 Ohara, Kamifukuoka-shi, Saitama 356-8502, Japan

\begin{abstract}
:
We propose a new method to characterize the conversion efficiency from the injected carriers to the excess photons in the process of all-optical gating in SOA, and investigated the efficiencies of SOAs with different structures.

(a)2006 Optical Society of America
\end{abstract}

OCIS codes: (250.5980) Semiconductor optical amplifiers; (190.5970) Semiconductor nonlinear optics including MQW;

\section{Introduction}

Semiconductor Optical Amplifiers (SOAs) are expected to perform essential function in future OTDM-WDM networks acting as ultrafast nonlinear optical devices. Demonstrations of all-optical demultiplexing, 3R regeneration and wavelength conversion using SOA-based all-optical gates have been reported for operation bit rates exceeding 100 Gbit/s[1-3]. As their capability of ultrafast gating has become clear, the lower limit of electric power consumption and its origin have been important issues from a physical and design viewpoint. When we use an SOA for optical gating, we must inject carriers into its active region at the cost of some electric power. Then population inversion is achieved, and we can cause stimulated emission, carrier recombination and gain/phase modulation by injecting cw or pulsed light. We have speculated, however, that there are several loss processes for the injected carriers, and only a part of them can be converted to photons through the stimulated emission. Then the conversion efficiency in each loss process will determine the required amount of power consumption. As far as we know, no method to evaluate these conversion efficiencies has been studied for SOA in an ultrafast operation. Therefore we propose a new characterization method of these efficiencies, and report measured results on several SOA samples with different chip-lengths and structures. This is our first step to find the way to fabricate an SOA with low power consumption.

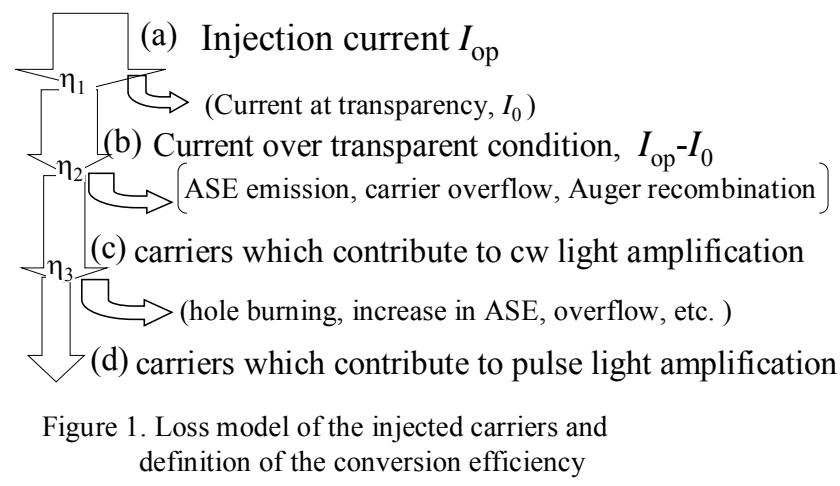

\section{Definition of carrier conversion efficiency in this work}

In this section we define the carrier conversion efficiencies, which will be used for the evaluation of the carrier losses in the SOA. Our loss model of the injected carrier is summarized in Fig. 1. The SOA stores carriers $N\left(I_{\mathrm{op}}\right)$ in its active region with the injection of a driving current $I_{\text {op }}$. When $I_{\text {op }}$ exceeds the transparent current $I_{0}$, (and $N$ exceeds $N_{0}$,) 


\section{OTuC4.pdf}

population inversion occurs and the SOA has a positive gain $\mathrm{G}(N)>1$. Thus we define $\eta_{1} \equiv\left(I_{\mathrm{op}}-I_{0}\right) / I_{\mathrm{op}}$ a current ratio which exceeds transparent current. Then the total number of the excess carriers, i.e. $N^{\mathrm{ex}} \equiv N-N_{0}$, can be approximately given as follows: In many applications, carrier dynamics in the SOA is well described with a rate equation model[4];

$$
\frac{d}{d t} \overline{n_{c}(t)}=\frac{I_{o p}}{q V}-\frac{\overline{n_{c}(t)}}{\tau_{c}}-\frac{1}{V} \cdot\left\{G\left[\overline{n_{c}(t)}\right]-1\right\} \cdot \frac{\left|E_{C W}\right|^{2}+\left|E_{\text {pulse }}(t)\right|^{2}}{\hbar \omega} .
$$

Where $n_{\mathrm{c}}$ stands for the carrier density averaged over the whole length of the active region (with volume $V$ ), $q$ represents the elemental charge, $E_{\mathrm{cw}}$ and $E_{\text {pulse }}$ are the amplitudes of the input lights and $\tau_{\mathrm{c}}$ is the carrier lifetime. When there is no input light, equation (1) has a steady solution. Then the excess carrier number $N^{\text {ex }}$ is given by

$$
N_{\text {injection }}^{e x}=\frac{I_{o p}-I_{0}}{q} \times \tau_{c} .
$$

We define this quantity as the nominal injection carrier number.

Apart from the estimation from current injection, we can estimate carrier densities from amplification of a cw light or ultrafast pulses in the SOA. If there are $N_{\mathrm{cw}}$ carriers which contribute to the cw amplification, $N_{\mathrm{cw}}$ should be related to the saturation power $P_{\text {sat }}{ }^{\mathrm{cw}}$, the carrier lifetime $\tau_{\mathrm{c}}$ and the small signal gain (SSG) $G_{0}[4,5]$. Similarly, if there are $N_{\text {pulse }}$ carriers which contribute to the pulse amplification, the saturation energy $E_{\text {sat }}{ }^{\text {pulse }}$ should be determined by them;

$$
\begin{aligned}
& N_{c w}=\frac{P_{s a t}^{c w}}{h v} \times \ln G_{0} \times \tau_{c}, \\
& N_{p u l s e}=\frac{E_{s a t}^{\text {pulse }}}{h v} \times \ln G_{0 .} .
\end{aligned}
$$

As illustrated in Fig. 1, we assume that $N_{\mathrm{cw}}$ is smaller than $N^{e x}$ injection due to ASE emission, carrier overflow, Auger recombination $e t c$., and that $N_{\text {pulse }}$ is smaller than $N_{\mathrm{cw}}$ due to spectral hole-burning etc. Then $\eta_{2} \equiv N_{\mathrm{cw}} / N^{e x}$ injection and $\eta_{3} \equiv N_{\text {pulse }} / N_{\text {cw }}$ represent the efficiencies in these loss processes.

For the ultrafast all-optical gating it is important to achieve large $N_{\text {pulse. }}$. That is realized when the total conversion efficiency $\eta \equiv \eta_{1} \eta_{2} \eta_{3}$ is maximized. It will be possible by improving each contribution separately, once their dominant factors are revealed.

\section{Conversion efficiency measurement and results}

To demonstrate the validity and utility of the former model, we performed SOA-parameter measurements and deduced carrier conversion efficiencies. So far we have measured seven SOA samples, as summarized in Table 1, to observe SOA-structure dependence. Two samples (K\#1 and $\mathrm{K \# 2}$ ) were designed by us and had similar cross-sectional structures with each other. The rest were commercial modules or custom chips by other manufacturers.

To obtain ultrafast optical pulses with lower frequency than carrier-recovery rates of the SOAs, we used a mode-locked fiber laser (MLFL: Pritel Inc., UOC-3) with a $\mathrm{LiNbO}_{3}$ modulator. Pulses with 2-ps width, $\lambda=1555 \mathrm{~nm}$ and $10.5-\mathrm{GHz}$ frequency from the MLFL were modulated down to $0.65-\mathrm{GHz}$ or $1.3-\mathrm{GHz}$ pulse train with the extinction ratio of typically $20 \mathrm{~dB}$. The carrier lifetimes for several injection currents were acquired through cross gain modulation measurements, using a cross-correlator (Femtochrome Research Inc., FR-103XR). CW light ( $\lambda=1548 \mathrm{~nm},-10 \sim-20$ $\mathrm{dBm}$ ) from a DFB-LD was gain-modulated in the SOA by the low-frequency pulses. By assuming that the gain recovery profile $\mathrm{G}(t)$ is proportional to $\exp \left(g\left(N(t)-N_{0}\right)\right)$, we obtained the carrier lifetimes $\tau_{\mathrm{c}}$. Gain saturation and SSG for $\mathrm{cw}$ input and also for pulse input were measured using same light sources and spectrum analyzers. The measured result of pulse-gain saturation for K\#1 sample is shown in Fig. 2 as an example. The saturation energy $E_{\text {sat }}$ for the ultrafast pulses was obtained by fitting to the theoretical gain saturation formula[5]. The saturation power $P_{\text {sat }}$ for the $\mathrm{cw}$ light was obtained from cw output power at 3-dB gain suppression, by $P_{\text {sat }}=P_{3 \mathrm{~dB}} / \ln 2$ [5].

Then the three kinds of excess carrier numbers $N$ and densities $n_{c}$ were obtained from those parameters. The results for two SOA samples are shown in Fig. 3. We observed that the measured carrier densities for all the SOA samples followed the relation: $n_{\text {injection }}^{e x} n_{\text {cw }}>n_{\text {pulse. }}$. This fact supports our carrier-loss model in Fig. 1 . 


\section{OTuC4.pdf}

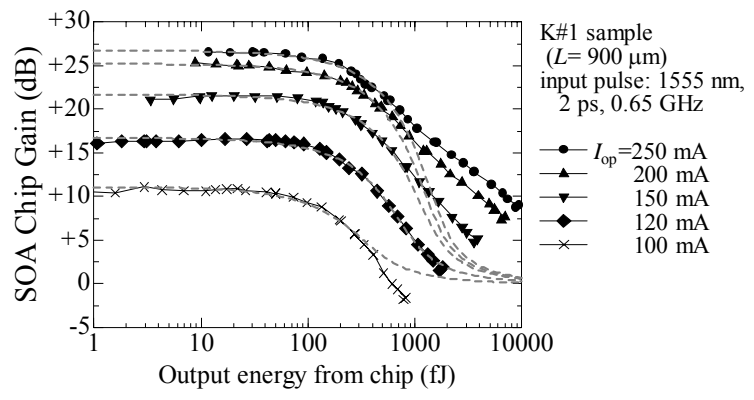

Figure 2: Example of measured gain saturation for pulse amplification. Dashed lines are fit curves.

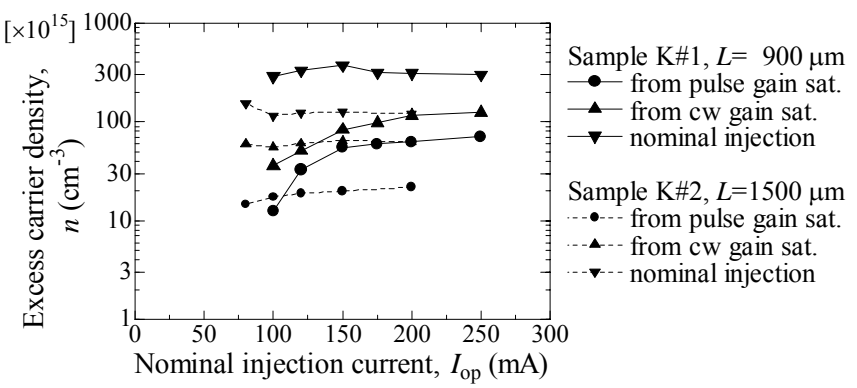

Figure 3: Measured results of the carrier densities at each stage in the Fig.1. $n_{\text {injection }}^{e x}$ : Fig.1(b), $n_{\text {cw }}:$ Fig. 1(c), $n_{\text {pulse }}:$ Fig. 1(d) (a)

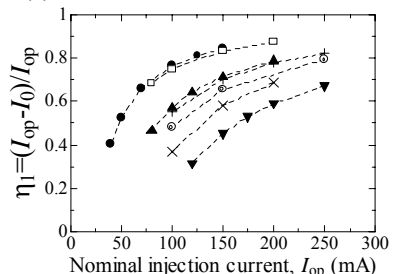

(c)

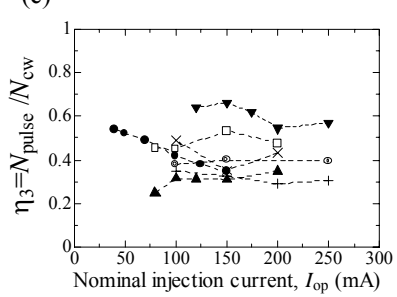

(b)

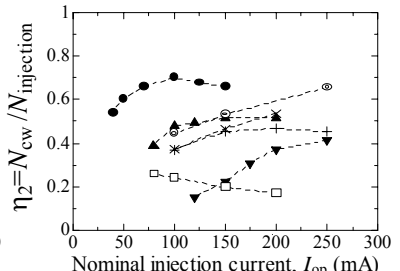

(d)

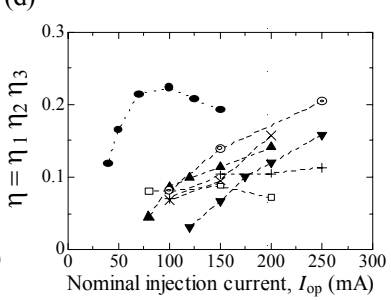

Table 1: list of the SOA samples

\begin{tabular}{|c|c|c|c|}
\hline Sample & Description & $\begin{array}{l}\text { Active Region } \\
\text { Length }(\mu \mathrm{m})\end{array}$ & Symbol \\
\hline $\mathrm{K} \# 1$ & custom & 900 & $-\nabla-$ \\
\hline $\mathrm{K} \# 2$ & custom & 1500 & $-\Delta-$ \\
\hline Av\#1 & $\begin{array}{l}\text { Avanex Inc., } \\
\text { A1901 }\end{array}$ & 700 & $-0-$ \\
\hline In\#1 & $\begin{array}{l}\text { InPhenix Inc., } \\
\text { custom chip }\end{array}$ & 300 & $-\square-$ \\
\hline $\mathrm{In} \# 2$ & $\begin{array}{l}\text { InPhenix Inc., } \\
\text { custom chip }\end{array}$ & 700 & -+- \\
\hline In\#3 & $\begin{array}{l}\text { InPhenix Inc., } \\
\text { custom chip }\end{array}$ & 1000 & $-x-$ \\
\hline $\operatorname{In} \# 4$ & $\begin{array}{l}\text { InPhenix Inc., } \\
\text { IPSAD1501 }\end{array}$ & 700 & $-0-$ \\
\hline
\end{tabular}

Figure 4: measured carrier-conversion efficiencies for each SOA samples.

(a), (b), (c): each efficiencies $\eta_{1} \sim \eta_{3}$ and (d): total efficiency

The conversion efficiencies in each of the loss processes are shown in Fig. 4. The total efficiencies $\eta$ of these SOA for the ultrafast gating use were around $0.1 \sim 0.2$. These results indicate that the total efficiency is not quite sensitive to the chip length, while largest $\eta_{3}$ and smallest $\eta_{2}$ were obtained with shortest samples in the set of simillar cross-sectional structure. In our next plan of surveying SOA series with different structures, we will search for the dominant factors for each efficiency and more efficient SOA structures.

\section{Conclusion}

We developed a new technique to characterize the conversion efficiencies of injected carriers into the carriers used in the ultrafast gating. This method was applied to SOA samples with different chip-lengths and structures. The results support our carrier-loss model. The conversion efficiency obtained in this work ranged from 0.1 to 0.2 . Our on-going research with this technique will provide a way to design and fabricate highly efficient SOA.

\section{References}

1. S. Nakamura, Y. Ueno, K. Tajima, "Error-free all-optical demultiplexing at $336 \mathrm{~Gb} / \mathrm{s}$ with a hybrid-integrated symmetric-Mach-Zehnder switch", Optical Fiber Communications Conference 2002, FD3.

2. Y. Ueno, S. Nakamura, and K. Tajima, "Penalty-Free Error-Free All-Optical Data Pulse Regeneration at 84 Gb/s by Using a Symmetric-Mach-Zehnder-Type Semiconductor Regenerator”, IEEE Photonics. Technol. Lett., vol. 13, 469-471, (2001).

3 . S. Nakamura, Y. Ueno, and K. Tajima, “168-Gb/s All-Optical Wavelength Conversion With a Symmetric-Mach-Zehnder-Type Switch”, IEEE Photonics. Technol. Lett., vol. 13, 1091-1093, (2001).

4. Y. Ueno, S. Nakamura, and K. Tajima, "Nonlinear phase shifts induced by semiconductor optical amplifiers with control pulses at repetition frequencies in the 40-160-GHz range for use in ultrahigh-speed all-optical signal processing”, J .Opt. Soc. Am. B vol. 19, 2573-2589 (2002).

5. T. Saitoh and T. Mukai, "Gain Saturation Characteristics of Traveling-Wave Semiconductor Laser Amplifiers in Short Optical Pulse Amplification”, IEEE J. Quantum. Electron., vol 26, 2086-2094, (1990). 